\title{
Deferiprone attenuates neuropathology and improves outcome following traumatic brain injury
}

\author{
Maria Daglas ${ }^{1}$, Phan Truong ${ }^{1}$, Linh Miles ${ }^{1}$, Sydney Juan ${ }^{1}$, Sonia Rao ${ }^{1}$, and Paul Adlard ${ }^{2}$ \\ ${ }^{1}$ The Florey Institute of Neuroscience and Mental Health \\ ${ }^{2}$ Florey Institute of Neuroscience and Mental Health
}

February 14, 2022

\begin{abstract}
Background and Purpose: Traumatic brain injury (TBI) remains a leading cause of mortality and morbidity in young adults. The role of iron in potentiating neurodegeneration following TBI has gained recent interest since iron deposition has been detected in the injured brain in the weeks to months post-TBI, in both the preclinical and clinical setting. A failure in iron homeostasis can lead to oxidative stress, inflammation and excitotoxicity; and whether this is a cause or consequence of the long-term effects of TBI remains unknown. Experimental approach: We investigated the role of iron, and the effect of therapeutic intervention using a brain-permeable iron chelator, deferiprone, in a controlled cortical impact mouse model of TBI. An extensive assessment of cognitive, motor and anxiety/depressive outcome measures were examined, and neuropathological and biochemical changes, over a 3-month period post-TBI. Key Results: Lesion volume was significantly reduced at 3 months, which was preceded by a reduction in astrogliosis and a preservation of neurons in the injured brain at 2 weeks and/or 1-month post-TBI in mice receiving oral deferiprone. Deferiprone treatment showed significant improvements in neurological severity scores and locomotor/gait performance, and cognitive function, and attenuated anxiety-like symptoms post-TBI. Deferiprone reduced iron levels, oxidative stress and altered expression of neurotrophins in the injured brain over this period. Conclusion and Implications: Our findings support a detrimental role of iron in the injured brain and suggest that deferiprone (or similar iron chelators) may be promising therapeutic approaches to improve survival, functional outcomes and quality of life following TBI.
\end{abstract}

Hosted file

BJP manuscript.pdf available at https://authorea.com/users/460381/articles/556408deferiprone-attenuates-neuropathology-and-improves-outcome-following-traumatic-braininjury 